\title{
Pattern of focal $\gamma$-bursts in chess players
}

\section{Grandmasters call on regions of the brain not used so much by less skilled amateurs.}

he brain's medial temporal lobe structures are thought to be important for the initial formation of long-term memory ${ }^{1,2}$, and active memory is indicated by bursts of $\gamma$-band activity in these and other areas of the association cortex ${ }^{3,4}$. Here we use a new technique of magnetic imaging to compare focal bursts of $\gamma$-band activity in amateur and professional chess players during matches. We find that this activity is most evident in the medial temporal lobe in amateur players, which is consistent with the interpretation that their mental acuity is focused on analysing unusual new moves during the game. In contrast, highly skilled chess grandmasters have more $\gamma$-bursts in the frontal and parietal cortices, indicating that they are retrieving chunks from expert memory by recruiting circuits outside the medial temporal lobe.

The 'chunking' theory of chess playing ${ }^{5}$
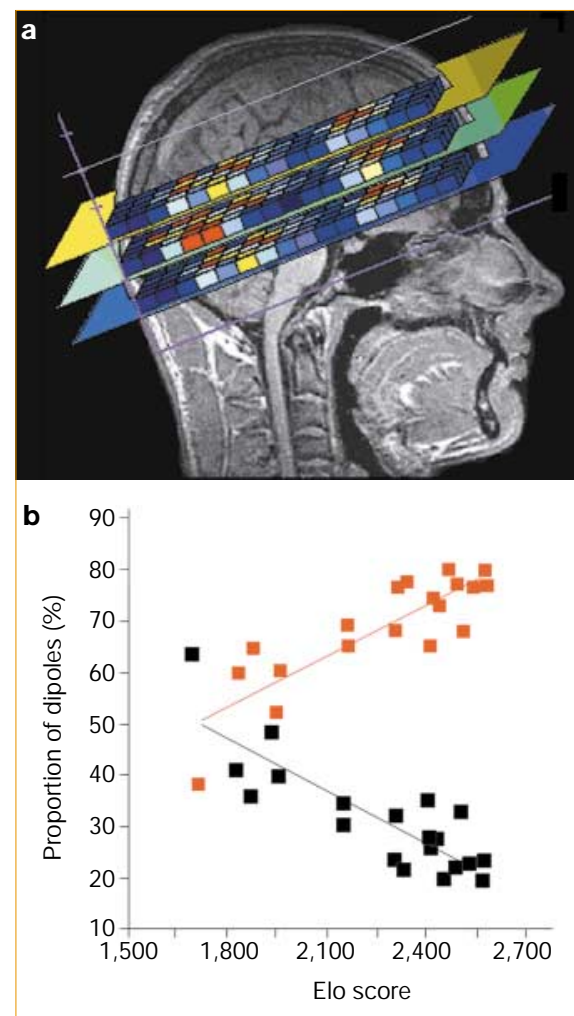

Figure 1 Focal $\gamma$-band activity in the brains of chess players. a, Determination of equivalent-current dipole density for inferior 'slices' through the hippocampus and medial temporal areas in an amateur player's brain. Increasing dipole density is indicated by a colour scale from dark to light blue, to yellow, to red. $\mathbf{b}$, Relationship between chess-playing skill (Elo rating scale) and the relative share of dipoles located in medial temporal lobe structures (black) and in the frontal and parietal cortices (red). Amateur players show more focal $\gamma$-bursts in the medial temporal lobe than grandmasters, who show more activity in the frontal and parietal cortices. suggests that expert memory is based on a large database of chunks in long-term memory. A chess grandmaster studies and practises for at least 10 years to learn more than 100,000 patterns (memory chunks). Consequently, grandmasters can 'recognize' the key elements in a problem situation much more rapidly than amateur players. Experts differ not only in the extent of their knowledge, but also in its organization. High-level processing elements, such as structuring knowledge and planning, assist in accessing the respective chunks ${ }^{6}$.

We tested 20 male players (aged $42 \pm 14$ years), each with more than 10 years of tournament and training practice. Ten professional grandmasters scored between 2,400 and 2,600 on Elo's chess-skill rating scale ${ }^{5}$; amateur players ranked 1,700 and above. Magnetoencephalographic recordings were made while subjects played against a computer and were scanned in the 5 seconds after each move by the computer program for focal $\gamma$-bursts $(20-40 \mathrm{~Hz}$; Fig. 1a). Examination of single slices indicates pronounced activity in the region of the perirhinal and entorhinal cortex, hippocampus and related structures in amateur players, but not in grandmasters.

There was a strong negative correlation $(r=-0.84)$ between the relative share of dipoles in these structures and Elo chess skill (Fig. 1b). The correlation was also significant when only the 12 players who had lost in the game were included in the analysis (draws and wins were achieved only by grandmasters). There was no relationship between the length or complexity of the game and the Elo score.
These marked differences in the distribution of focal brain activity during chess playing point to differences in the mechanisms of brain processing and functional brain organization between grandmasters and amateurs. Lesions in structures that are activated in amateur players impair recent memory while leaving remote memory intact ${ }^{2}$. Grandmasters seem to rely more on remote than on recent memory.

High-level processing elements ${ }^{6}$ may also have contributed to the observed differences. The chunking theory of memory states that the number and nature of chunks that chess experts can hold in long-term memory can be used to predict chess performance $^{6}$. Our results indicate that the activation of expert memory chunks produces focal $\gamma$-band activity in the neocortex, whereas amateur players primarily encode and analyse new information, tasks that activate the medial temporal lobe and the hippocampus. It is possible that these structures play only a transitional role during the establishment of expert memory in the neocortex.

Ognjen Amidzic, Hartmut J. Riehle, Thorsten Fehr, Christian Wienbruch, Thomas Elbert

University of Konstanz, Universitätsstrasse 10,

Fach-D 30, 78457 Konstanz, Germany

e-mail: ognjen.amidzic@uni-konstanz.de

1. Bontempi, B., Laurent-Demir, C., Destrade, C. \& Jaffard, R. Nature 400, 671-674 (1999).

2. Squire, L. R. Psychol. Rev. 99, 195-231 (1992)

3. Pulvermüller, F., Keil, A. \& Elbert, T. Trends Cogn. Sci. 3, 250-252 (1999).

4. Singer, W. et al. Trends Cogn. Sci. 1, 252-261 (1997).

5. Elo, A. E. The Rating of Chess Players, Past and Present (Arco, New York, 1978).

6. Gobet, F. Cognition 66, 115-152 (1998)
Konstanzer Online-Publikations-System (KOPS)

URL: http://www.ub.uni-konstanz.de/kops/volltexte/2007/4147/ URN: http://nbn-resolving.de/urn:nbn:de:bsz:352-opus-41475 